\title{
Searching for dark sectors in multi lepton final state in $e^{+} e^{-}$collisions
}

\author{
Paolo Ciafaloni, ${ }^{a}$ Gabriele Martelli ${ }^{b}$ and Mauro Raggi ${ }^{c}$ \\ ${ }^{a}$ INFN and Università del Salento, \\ Via per Arnesano, Lecce, Italy \\ ${ }^{b}$ Dipartimento di Fisica e Geologia, Università di Perugia, \\ Via Alessandro Pascoli, Perugia, Italy \\ ${ }^{c}$ Dipartimento di Fisica, Sapienza Università di Roma, \\ p.le Aldo Moro 2, Roma, Italy \\ E-mail: paolo.ciafaloni@le.infn.it, gabriele.martelli@pg.infn.it, \\ mauro.raggi@roma1.infn.it
}

ABSTRACT: Electron positron collisions are a very promising environment to search for new physics, and in particular for dark sector related observables. The most challenging experimental problem in detecting dark sector candidates is the very high associated Standard Model background. For this reason it is important to identify observables that are, at the same time, minimally suppressed in the dark sector and highly suppressed in the Standard Model. One example is the $e^{+} e^{-} \rightarrow 3\left(e^{+} e^{-}\right)$process that can be mediated either by the production and subsequent decay of dark Higgs $\left(h^{\prime}\right), e^{+} e^{-} \rightarrow A^{\prime} h^{\prime} \rightarrow 6 e$ [1] or produced by the Standards Model process $e^{+} e^{-} \rightarrow 3\left(e^{+} e^{-}\right)$. In the following letter we study the relative contribution to observed $e^{+} e^{-} \rightarrow 3\left(e^{+} e^{-}\right)$total cross section, coming from the $h^{\prime}$ mediated and from the Standard Model processes in the contest of fixed target and low energy collider experiments, with particular attention to the PADME experiment at the INFN Laboratori Nazionali di Frascati.

KEywords: Beyond Standard Model, Dark matter, Fixed target experiments, Lepton production

ArXiv EPrint: 2012.04754 


\section{Contents}

1 Introduction 1

$2 e^{+} e^{-} \rightarrow e^{+} e^{-} e^{+} e^{-}$in the equivalent photon approximation and beyond 2 2.1 Calculation of $\sigma_{e^{+} e^{-} \rightarrow e^{+} e^{-} e^{+} e^{-}}$using CalcHEP 4

2.2 Calculation of $\sigma_{e^{+} e^{-} \rightarrow e^{+} e^{-} e^{+} e^{-}}$using numerical EPA approximation 5

$3 e^{+} e^{-} \rightarrow e^{+} e^{-} e^{+} e^{-} e^{+} e^{-}$six leptons in the Equivalent Photon Approximation and beyond

4 Phenomenology of Dark Higgs at low energy 8

4.1 Dark Higgs production cross sections 8

4.2 SM 6-leptons and Dark Higgs decay comparison 9

$\begin{array}{lll}4.3 & \text { Background suppression } & 10\end{array}$

5 Multi lepton perspectives in PADME 11

6 Conclusions 12

A The Equivalent Photon Approximation (EPA) 13

\section{Introduction}

In recent years, the idea that dark matter can be considerably lighter than the weak scale and secluded by portal interactions, has attracted growing interest, supported by the very stringent limits on dark matter at $\mathrm{GeV}-\mathrm{TeV}$ mass scales obtained by direct detection and LHC experiments.

Among the possible different realisations of dark sectors involving portal interaction and new mediators, the kinetic mixing with $U(1)$ hypercharge is of considerable interest from a phenomenological point of view as the channel where hidden sector may be probed with maximal sensitivity.

The authors of [1] have investigated signatures of a minimal secluded $\mathrm{U}(1)_{D}$ extension of the SM at medium energy colliders while in [2] the dark group is non-abelian. The proposed models assume the existence, in addition to the $\mathrm{U}(1)_{D}$ "dark" photon $A^{\prime}$, of an elementary dark Higgs boson $\left(h^{\prime}\right)$, which spontaneously breaks the $\mathrm{U}(1)_{D}$ symmetry, and of dark matter particles with WIMP scale masses. One of the characteristics of this class of extended models, is the possibility of producing spectacular multi lepton pairs final states originating, from $h^{\prime}$ decay chain. This kind of processes have been described in different environments: in B physics [1, 2], in charm physics [3], and more recently in kaon decays [4]. 
At $e^{+} e^{-}$colliders, the amplitude of the dominant $h^{\prime}$ production mechanism, the so called Higgs'-strahlung $e^{+} e^{-} \rightarrow A^{\prime} h^{\prime}$ [1], is minimally suppressed in the dark sector by just a single power of the kinetic mixing parameter $\epsilon$. Under the assumption that both $m_{h^{\prime}}$ and $m_{A^{\prime}}$ are below the di-muon threshold $210 \mathrm{MeV}$, at the end of the decay chains of both $h^{\prime}$ and $A^{\prime}$ we will only find SM particles. The $A^{\prime}$ decay will produce an $e^{+} e^{-}$pair while the $h^{\prime}$ decay will produce to $2\left(e^{+} e^{-}\right)$, leading to a minimally suppressed final state of $3\left(e^{+} e^{-}\right)$. On the contrary the Standard Model process $e^{+} e^{-} \rightarrow 3\left(e^{+} e^{-}\right)$suffers a strong $\alpha^{6}$ suppression, which can compensate the $\epsilon^{2}$ suppression of the dark sector rate.

Experiments at $e^{+} e^{-}$colliders with few $\mathrm{GeV}$ center of mass energy and very high luminosity, Babar [5] and Belle [6], had investigated $e^{+} e^{-} \rightarrow 3\left(\ell^{+} \ell^{-}\right)$setting stringent limits on the existence of the $h^{\prime}$ for energy scales in the $\sim \mathrm{GeV}$ range. No data are available for masses of the $h^{\prime}<1 \mathrm{GeV}$ which can be probed by low energy fixed target experiments.

For this reason it is worth investigating the scale of the contribution of dark sector particles decays to the cross section of the process $e^{+} e^{-} \rightarrow 3\left(e^{+} e^{-}\right)$, compared to Standard Model one at low energy colliders. Being the dark contribution in any case small, in this paper we will develop new techniques to precisely calculate the SM contribution.

The paper is organised as follows: in section $2(3)$ we discuss the various approximations for the $e^{+} e^{-} \rightarrow 2\left(e^{+} e^{-}\right)\left(e^{+} e^{-} \rightarrow 3\left(e^{+} e^{-}\right)\right)$process. In section 4 we introduce the Dark Higgs Model which is our reference one in this paper, and we compare $e^{+} e^{-} \rightarrow 3\left(e^{+} e^{-}\right)$ cross sections in this model and in the Standard Model. In section 5 we specialise the discussion to the PADME experiment; our conclusions are drawn in section 6. Finally, appendix A is devoted to the Equivalent Photon Approximation (EPA) which is used throughout the paper.

In the following we refer to $e^{+} e^{-} \rightarrow 2\left(e^{+} e^{-}\right)$as the " $4 \ell$ process" and to $e^{+} e^{-} \rightarrow$ $3\left(e^{+} e^{-}\right)$as the " $6 \ell$ process".

\section{$2 e^{+} e^{-} \rightarrow e^{+} e^{-} e^{+} e^{-}$in the equivalent photon approximation and be- yond}

Due to the large number of Feynman diagrams involved, there is no exact analytic expression for the $\mathrm{QED}^{1}$ tree level cross section for $e^{+} e^{-} \rightarrow 2\left(e^{+} e^{-}\right)$, nor for $e^{+} e^{-} \rightarrow 3\left(e^{+} e^{-}\right)$. Therefore, one has to make use to various kinds of approximations, that follow three main lines:

- The Equivalent Photon Approximation (EPA) [7], similar to the parton model approach in QCD, which is described in detail in appendix A.

- Tree level numerical calculations; here we perform such calculations making use of the CalcHEP tool [10].

- Approximate analytic expressions [7], which retain the leading term in an expansion in the small parameter $m^{2} / s, m$ being the electron mass and $\sqrt{s}$ the c.m. energy.

\footnotetext{
${ }^{1}$ At the energies considered here and for the given processes, the QED sector of the Standard Model is the only relevant one.
} 
In the latter case, the analytic approximation has the form:

$$
\sigma=\frac{1}{m^{2}}\left(a_{n} \log ^{n} l^{n}+a_{n-1} l^{n-1}+\cdots+a_{0}\right)+\mathcal{O}\left(\frac{1}{s}\right)
$$

where $n=3(n=4)$ for the $4 \ell(6 \ell)$ process. The EPA allows to calculate the first term in the series, with the highest power of $l$; we call this the 'Leading Log EPA' (LLEPA) and we discuss how to obtain this approximation in appendix A.

In the literature there are different analytical formulae which allow to compute the total cross section for the SM process $e^{+} e^{-} \rightarrow 2\left(e^{+} e^{-}\right)$. We will start considering the calculation using the Equivalent Photon Approximation (EPA), and then compare it to more accurate calculation which are possible in the $4 \ell$ case. According to a classical paper dated back to $1975[7]$ the total cross section $e^{+} e^{-} \rightarrow e^{+} e^{-} e^{+} e^{-}$using equivalent photon approximation at leading log reads:

$$
\sigma_{e^{+} e^{-} \rightarrow e^{+} e^{-} e^{+} e^{-}} \approx \frac{28 \alpha^{4}}{27 \pi m_{e}^{2}}\left(\log \frac{s}{m_{e}^{2}}\right)^{3}
$$

where $s$ is the center of mass energy squared that for a fixed target experiment is $s \sim$ $2 E_{\text {beam }} m_{e}$. Equation (2.2) only includes the leading logarithmic term of the cross section while more accurate calculation can be obtained analytically. The paper by Budnev et al. [7] also contains, in its appendix F1, a complete analytical calculation of the cross section in which all the coefficients for the lower order logarithmic terms are worked out.

$$
\sigma_{e^{+} e^{-} \rightarrow e^{+} e^{-} e^{+} e^{-}}=\frac{28 \alpha^{4}}{27 \pi m_{e}^{2}}\left(\ell^{3}-A \ell^{2}+B \ell+C\right)+O\left(\frac{1}{s}\right) ; \quad \ell=\log \frac{s}{m_{e}^{2}}
$$

where $\ell$ is the logarithmic term in eq. (2.2). For electron-positron scattering the coefficients $\mathrm{A}, \mathrm{B}, \mathrm{C}$ have been calculated, neglecting interference between produced and scattered electrons, to be [7]:

$$
A=178 / 28 \sim 6.36 \quad B \sim-11 \quad C \sim 100
$$

Looking at equation (2.3), it appears that in fixed target experiment, in which $s$ is typically small and the $\ell$ term ranges from 7-10, none of the logarithmic term is negligible due to the high values of coefficients A, B and C. At $e^{+} e^{-}$colliders like PEP-II, KEKB and SuperKEKB, where $s$ can reach the $10 \mathrm{GeV}$ range and the log term the value of 20 , the $\ell^{2}$ terms still accounts for $\sim 30 \%$ of the total cross section.

Performing a numerical analysis we observe that, in particular at low energy colliders, accidental cancellations are almost complete among the dominant $\ell$ terms. At the energy scale of the PADME experiment $\left(E_{B} \sim 500 \mathrm{MeV}\right)$ we obtain:

- $\frac{28 \alpha^{4}}{27 \pi m_{e}^{2}} \ell^{3} \sim 6.0 \times 10^{8} \mathrm{pb}$

- $\frac{28 \alpha^{4}}{27 \pi m_{e}^{2}}\left(-A \ell^{2}\right) \sim-5.0 \times 10^{8} \mathrm{pb}$ 


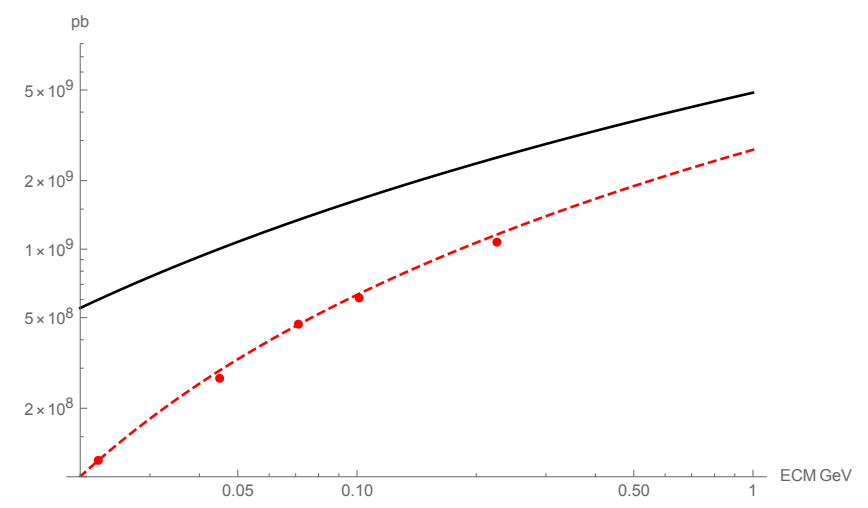

Figure 1. Comparison of $\sigma_{e^{+} e^{-} \rightarrow e^{+} e^{-} e^{+} e^{-}}$at fixed target CM energies computed with eq. (2.3), dashed red line, with CalcHEP, red dots, and with Leading Log EPA eq. (2.2), black line.

$$
\begin{aligned}
& \text { - } \frac{28 \alpha^{4}}{27 \pi m_{e}^{2}}(B \ell) \sim-1.16 \times 10^{8} \mathrm{pb} \\
& \text { - } \frac{28 \alpha^{4}}{27 \pi m_{e}^{2}}(C) \sim 1.4 \times 10^{8} \mathrm{pb}
\end{aligned}
$$

In this energy regime the sum of the three logarithmic terms of eq. (2.3) almost cancels out and only accounts for $\sim 15 \%$ of the total cross section, which is surprisingly dominated by the constant term. For this reason is crucial to check, in the absence of experimental results to compare with, if the coefficients $\mathrm{A}, \mathrm{B}$, and $\mathrm{C}$ of eq. (2.3) have been correctly calculated.

\subsection{Calculation of $\sigma_{e^{+} e^{-} \rightarrow e^{+} e^{-} e^{+} e^{-}}$using CalcHEP}

In order to check the reliability of eq. (2.3) we have performed a tree level numerical calculation of the cross section of the process $e^{+} e^{-} \rightarrow 2\left(e^{+} e^{-}\right)$based on the CalcHEP tool [10]. To improve the precision of the calculation, the mass of the electron was introduced in the CalcHEP model and the value of the electromagnetic constant $\alpha$ adapted to low energy scales. The contributions coming from diagrams mediated by the $\mathrm{Z}$ bosons, which have been verified using CalcHEP to be smaller than $1 \times 10^{-4}$, are neglected in the calculations.

In figure 1 the comparison is shown for different values of the center of mass energy corresponding to fixed target experiments at different possibile extracted positron beam lines. The first point corresponds to the DA $\Phi$ NE Linac beam energy $0.5 \mathrm{GeV}$, the third point corresponds to the energy of an extracted positrons beam from the Cornell Electron Storage Ring (CESR $5 \mathrm{GeV}$ ), the fourth point, beam energy $\sim 10 \mathrm{GeV}$, corresponds to the proposed JLab positron facilities at the CEBAF accelerator [8], while the last one at $50 \mathrm{GeV}$ a positron beam produced by the North area facilities at CERN. The plot show that a very good agreement, to the level of few \%, is achieved at any energy in between the fully analytical cross section and the CalcHEP based tree level calculation, while the leading log EPA approximation is inaccurate by factors, from 5 to 2 . This huge discrepancy below the $\mathrm{GeV}$ scale originates from an accidental cancellation between the $\ell^{3}$ and the $\ell^{2}$ terms in eq. (2.3); moreover, the constant term is $\sim 100$. Of course, at higher energies the 


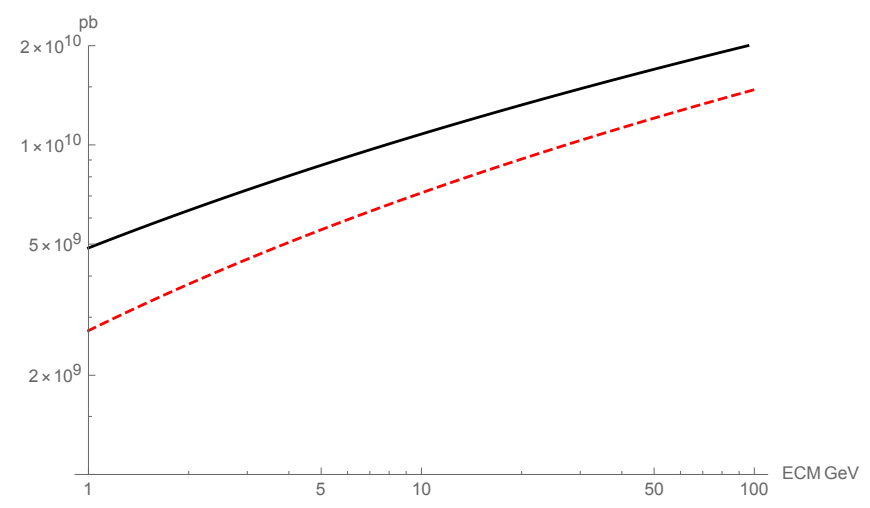

Figure 2. Comparison of $\sigma_{e^{+} e^{-} \rightarrow e^{+} e^{-} e^{+} e^{-}}$at collider CM energies computed with eq. (2.3), dashed red line, and with leading log EPA eq. (2.2), black line.

$\ell^{3}$ term starts to dominate and the Leading Log EPA becomes a better approximation of the analytic expression.

In figure 2 the comparison is shown for different $s$ values relevant for collider experiments from KLOE at DA $\Phi N E, 1 \mathrm{GeV}$, to the energy scale of the experiments at the CERN LEP collider. In this regime the overestimate of the cross section value due to the Leading Log EPA approximation, 2, appears to be more stable over a large range of energy, as the cross section also is. To conclude, the leading log EPA provides a poor approximation of the analytic expression for the cross section of the $4 \ell$ process due to accidental cancellations.

\subsection{Calculation of $\sigma_{e^{+} e^{-} \rightarrow e^{+} e^{-} e^{+} e^{-}}$using numerical EPA approximation}

As shown in the previous paragraph the precision of the leading log EPA approximation in predicting the value of the $\sigma_{e^{+} e^{-} \rightarrow e^{+} e^{-} e^{+} e^{-}}$is poor, in particular at energies below the $\mathrm{GeV}$. For this reason we developed an alternative approach which allows to maintain better precision, without performing a full tree level calculation as we did with CalcHEP. This

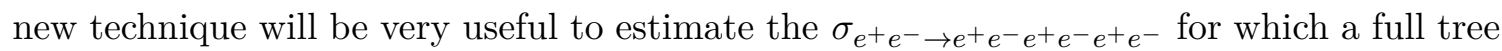
level calculation cannot be achieved.

Let us summarise the EPA with the following expression (A.5):

$$
\sigma(s)=\int_{\epsilon}^{1} d \tau \mathcal{L}(\tau) \sigma_{p}(\tau s) \quad \mathcal{L}(\tau)=\int_{\tau}^{1} \frac{d x}{x} f_{\gamma e}(x) f_{\gamma e}\left(\frac{\tau}{x}\right)
$$

In the leading $\log$ EPA we approximate $f_{\gamma e}(x)$ and $\mathcal{L}(\tau)$ in order to obtain the leading log value for the cross section, as explained in the appendix. However we can instead retain the full expressions for the splitting functions and the luminosity, thus improving the approximation. The full expression for the splitting function can be obtain by integrating in $\boldsymbol{k}_{\perp}^{2}$ the expression given in eq. (5.18) of [7] from $\left|\boldsymbol{k}_{\perp}^{2}\right|_{\min }=x^{2} m^{2}$ to $\left|\boldsymbol{k}_{\perp}^{2}\right|_{\max }=m^{2}$ to produce:

$$
f_{\gamma e}(x)=\frac{\alpha}{2 \pi}\left(\frac{1+(1-x)^{2}}{x} \log \frac{1}{x^{2}}-(1-x)\left(1-x^{2}\right)\right)
$$




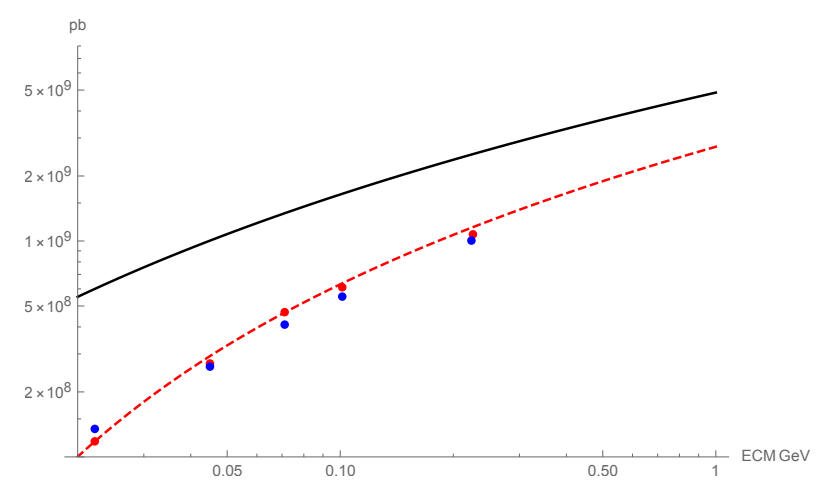

Figure 3. Comparison of $e^{+} e^{-} \rightarrow 2\left(e^{+} e^{-}\right)$at fixed target CM energies. Analytic calculations: full EPA eq. (2.3), dashed red line, LLEPA eq. (2.2), black line. Numerical calculations: Calchep, red dots, "numerical EPA", blue dots.

while the expression for the cross section $\gamma \gamma \rightarrow e^{+} e^{-}$can be found in (E.4) of [7]:

$$
\sigma_{\gamma \gamma}=\frac{4 \pi \alpha^{2}}{s \tau}\left(1+4 \frac{m^{2}}{s \tau}-8 \frac{m^{4}}{s^{2} \tau^{2}} L-\left(\frac{1}{\tau}+4 \frac{m^{2}}{s \tau^{2}}\right) t\right)
$$

with

$$
L=2 \log \left[\frac{1}{2} \sqrt{\frac{\tau s}{m^{2}}}+\sqrt{\frac{\tau s}{4 m^{2}}-1}\right], \quad t=\tau \sqrt{1-4 \frac{m^{2}}{s \tau}}
$$

Expressions (2.6), (2.7), (2.8) can be fetched into (2.5) in order to obtain the cross section value for the $e^{+} e^{-} \rightarrow 2\left(e^{+} e^{-}\right)$process. The price we pay is that it is no longer possible to obtain an analytic expression, and the cross section is evaluated numerically: we call this approximation "numerical EPA". Contrarily to the leading log EPA, the numerical cross section is a much better approximation for the tree level cross section. This can be seen in figure 3, where we can see that the numerical EPA approximation (blue dots) is very close to both the CalcHEP numerical value (red dots) and to the analytic approximation of eq. (2.3). The numerical EPA approximation will be very useful for the $6 \ell$ case (see section 3), where no numerical calculation can be performed and no analytic approximation was available before the writing of this paper.

\section{$3 e^{+} e^{-} \rightarrow e^{+} e^{-} e^{+} e^{-} e^{+} e^{-}$six leptons in the Equivalent Photon Approx- imation and beyond}

The cross section of the SM process $e^{+} e^{-} \rightarrow 3\left(e^{+} e^{-}\right)$is very hard to calculate due to the high number of particle in the final states producing several thousand of Feynman diagrams which contributes to the amplitude. Our attempt to obtain a numerical result for the cross section failed using both CalcHEP [10] and MadGraph [11] tools. A formula to estimate the order of magnitude of the cross section is proposed in eq. 12 of [1]:

$$
\sigma_{e^{+} e^{-} \rightarrow 3\left(e^{+} e^{-}\right)} \approx \frac{\alpha^{6}}{\pi^{3} m_{e}^{2}}\left(\log \frac{s}{m_{e}^{2}}\right)^{4}
$$




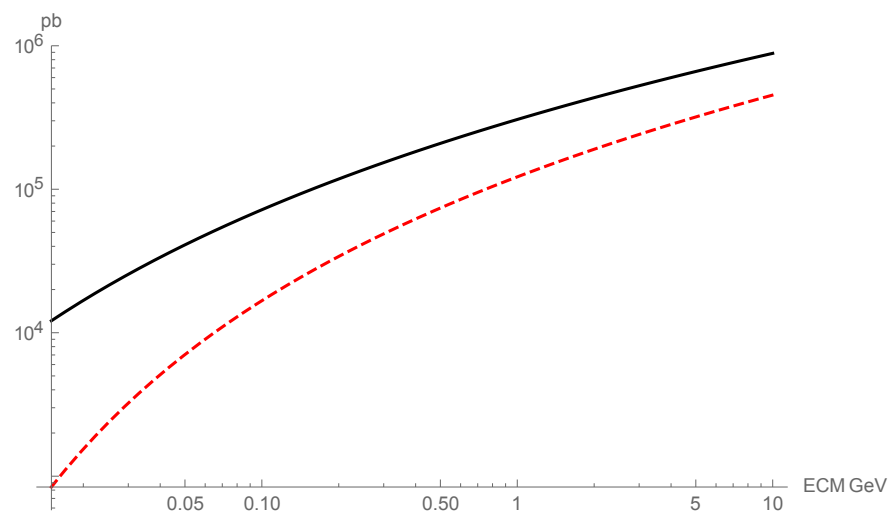

Figure 4. Leading Log EPA (in black) and Numerical EPA (red dashed) approximations for the tree level $e^{+} e^{-} \rightarrow e^{+} e^{-} e^{+} e^{-} e^{+} e^{-}$cross section.

A slightly more precise estimate can be obtained by using the Leading Log EPA approximation by computing the cross section $\sigma\left(\gamma \gamma \rightarrow e^{+} e^{-} e^{+} e^{-}\right)$as proposed by [7]:

$$
\sigma_{e^{+} e^{-} \rightarrow 3\left(e^{+} e^{-}\right)} \approx \frac{\alpha^{2}}{6 \pi^{2}} \sigma_{\left(\gamma \gamma \rightarrow e^{+} e^{-} e^{+} e^{-}\right)}\left(\log \frac{s}{m_{e}^{2}}\right)^{4}
$$

where $\sigma_{\left(\gamma \gamma \rightarrow e^{+} e^{-} e^{+} e^{-}\right)}$can be estimated using [12]. This expression coincides with (A.8), that we derived using Leading Log EPA. Like in the $4 \ell$ case, we expect eq. (3.2) to be a very poor approximation of the tree level cross section. Unlike the $4 \ell$ case, in the present literature there is no full analytic expression nor numerical calculation for the $6 \ell$ case. However we expect the Numerical EPA approximation to be a much better estimate of the tree level cross section than eq. (3.2).

Using full numerical integration we derived a new expression for the cross section for the process $e^{+} e^{-} \rightarrow 3\left(e^{+} e^{-}\right)$down to the constant term:

$$
\begin{aligned}
\sigma_{\text {EPAfull }}=\frac{\alpha^{2}}{6 \pi^{2}} \sigma_{\left(\gamma \gamma \rightarrow e^{+} e^{-} e^{+} e^{-}\right)}\left(\log ^{4}\left(\frac{s}{m^{2}}\right)\right. & +\mathrm{Alog}^{3}\left(\frac{s}{m^{2}}\right) \\
+ & \left.\mathrm{B} \log ^{2}\left(\frac{s}{m^{2}}\right)+\mathrm{C} \log \left(\frac{s}{m^{2}}\right)+\mathrm{D}\right)
\end{aligned}
$$

where the constant coefficients have the following values:

$$
A \sim-11.9 \quad B \sim 22.62 \quad C \sim 143.5 \quad D \sim-521.1
$$

Like in the case of eq. (2.3), accidental cancellation due to high values of the low power logarithmic term's constants is observed. In this case negative high values of the constants $A$ and $D$, compared to the $\log$ scaling factor at low energies $<10$, strongly reduce the value of the total cross section, compared to the leading log term estimate. In figure 4 the Leading Log EPA, eq. (3.2), and the numerical EPA cross sections, eq. (3.3), are compared as function of the center of mass energy; like in the $4 \ell$ case, the leading log expression grossly overestimates the tree level cross section at low CM energies, while the disagreement decreases at high energy due to increased value of the Log term. A key ingredient in the absolute value of the cross section common to the two parameterisation is the $\sigma_{(\gamma \gamma \rightarrow 4 \ell)}$, assumed to be constant as function of the CM energy [7]. 


\section{Phenomenology of Dark Higgs at low energy}

The authors of [1] consider a minimal extension of the SM by adding to the SM a $\mathrm{U}(1)_{D}$ gauge boson $A^{\prime}$ and a single complex scalar Higgs' field $\phi$ responsible for spontaneous symmetry breaking.

They assume that any additional particles, in particular possible dark matter candidates, are heavy compared to the $A^{\prime}$ and $h^{\prime}$ mass scales. All interactions with the SM and the secluded sector proceed through kinetic mixing of $\mathrm{U}(1)_{D}$ with the Standard Model photon. We can neglect mixing with the $\mathrm{Z}$ boson and Standard Model Higgs boson which will be irrelevant at the energy scale under scrutiny for this paper.

The Lagrangian containing the physical Higgs' field $h^{\prime}$ takes the form:

$$
\mathcal{L}=-\frac{1}{4} A_{\mu \nu}^{2}+\frac{1}{2} m_{A^{\prime}}^{2}+\frac{1}{2}\left(\partial_{\mu} h^{\prime}\right)^{2}++\frac{1}{2} m_{h^{\prime}}^{2} h^{\prime 2}+\mathcal{L}_{\text {int }}
$$

With respect to the rich phenomenology described in [1] for center of mass energy below the di-muon threshold, the situation is much simplified. Neglecting the possible decay to neutrinos, which is extremely suppressed at this energy, the only possible $A^{\prime}$ decay is in $e^{+} e^{-}$pairs. The decay width has the form:

$$
\Gamma_{A^{\prime} \rightarrow e e}=\frac{1}{3} \alpha \epsilon^{2} m_{A^{\prime}} \sqrt{1-\frac{4 m_{e}^{2}}{m_{A^{\prime}}^{2}}}\left(1+\frac{2 m_{e}^{2}}{m_{A^{\prime}}^{2}}\right)
$$

and for low energy and $\epsilon>1 \times 10^{-4}$ the decay $A \rightarrow e^{+} e^{-}$is prompt.

Concerning the $h^{\prime}$ decays only few possibilities exist. If the mass hierarchy is such that $m_{h^{\prime}}>2 m_{A^{\prime}}$, the dominant decay is to a pair of on shell $A^{\prime}\left(h^{\prime} \rightarrow A^{\prime} A^{\prime} \rightarrow 2\left(e^{+} e^{-}\right)\right)$. In this scenario the decay is always prompt because the lifetime only depends on $\alpha_{D}$ which is supposed to be large compared to $\epsilon$. For masses $m_{h^{\prime}}<2 m_{A^{\prime}}$ decays to off-shell $A^{\prime}$ are also possible but, due to the suppression of the 4 body decay $h^{\prime} \rightarrow A^{\prime} * A^{\prime} * \rightarrow 2\left(e^{+} e^{-}\right)$, the dominant decay proceeds through triangle graphs to an $e^{+} e^{-}$pair [1].

$$
\tau\left(h^{\prime} \rightarrow e^{+} e^{-}\right) \sim \frac{\alpha_{D} \alpha^{2} \epsilon^{4} m_{h^{\prime}}}{2 \pi^{2}} \frac{4 m_{e}^{2}}{m_{A^{\prime}}^{2}}
$$

The lifetime in this case is extremely long and the Dark Higgs will be stable on the experiment scale producing missing energy in the final state. Under the muon production threshold the possible dark sector final states induced by $h^{\prime}$ produced in association with an $A^{\prime}$ are either $3\left(e^{+} e^{-}\right)$or $\left(e^{+} e^{-}\right)+$missing energy.

\subsection{Dark Higgs production cross sections}

One of the few $h^{\prime}$ production processes is the so-called Higgs-strahlung, $e^{+} e^{-} \rightarrow h^{\prime} A^{\prime}$, which has an amplitude that is suppressed by just a single power of the kinetic mixing angle and can therefore readily occur for $\epsilon \sim O\left(10^{-2}-10^{-3}\right)$. This production mechanism is similar to traditional Higgs-strahlung in the SM but in this case the $h^{\prime}$ is produced in association with a $A^{\prime}$ instead of a SM photon. The total cross section for the Higgs-strahlung process 


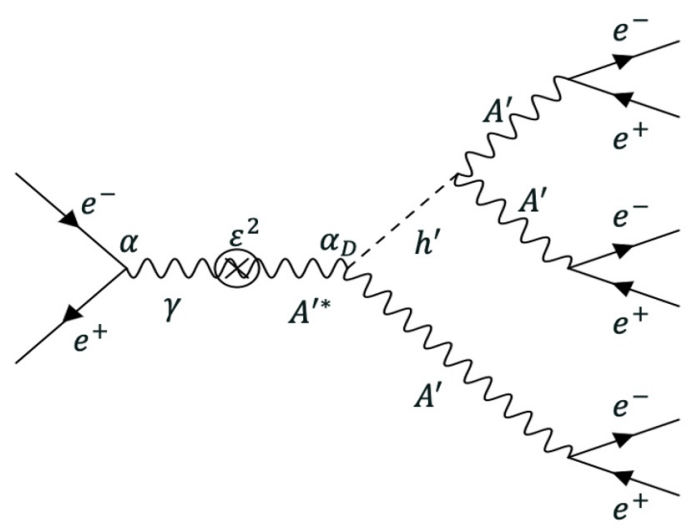

Figure 5. Feynman diagram for the Higgs-strahlung process producing a six leptons final state.

reads [1]:

$$
\begin{aligned}
\sigma_{e^{+} e^{-} \rightarrow A^{\prime} h^{\prime}}= & \frac{\pi \alpha \alpha_{D} \epsilon^{2}}{3 s}\left(1-\frac{m_{A^{\prime}}^{2}}{s}\right)^{-2} \sqrt{\lambda\left(1, \frac{m_{h^{\prime}}^{2}}{s}, \frac{m_{A^{\prime}}^{2}}{s}\right)} \\
& \times\left[\lambda\left(1, \frac{m_{h^{\prime}}^{2}}{s}, \frac{m_{A^{\prime}}^{2}}{s}\right)+\frac{12 m_{A^{\prime}}^{2}}{s}\right]
\end{aligned}
$$

where $\alpha_{D}$ is the coupling between $h^{\prime}$ and $A^{\prime}$ and where $\lambda(a, b, c) \equiv a^{2}+b^{2}+c^{2}-2 a b-$ $2 a c-2 b c$.

For reasonable values of the kinetic mixing parameter $\epsilon$, the cross section is quite large compared to the SM $6 \ell$ process. In fact the Higgs-strahlung cross section only pays an $\epsilon^{2} \alpha \alpha_{D}$ suppression compared to the $\alpha^{6}$ of the concurrent SM process. For fixed values of the $m_{h^{\prime}}$ and $m_{A^{\prime}}$ the production cross section scales as $1 / s$ which disfavour the production $\sim 10 \mathrm{MeV}$ scale particles at high energy colliders.

\subsection{SM 6-leptons and Dark Higgs decay comparison}

We pointed out that $e^{+} e^{-} \rightarrow 3\left(e^{+} e^{-}\right)$final state in $e^{+} e^{-}$collisions can be produced by two competing mechanisms: the beyond the SM process $e^{+} e^{-} \rightarrow h^{\prime} A^{\prime} \rightarrow 3\left(e^{+} e^{-}\right)$, shown in figure 5 and the Standard Model process $e^{+} e^{-} \rightarrow 3\left(e^{+} e^{-}\right)$. In this section we will estimate the relative contribution to experimental total cross section $e^{+} e^{-} \rightarrow 3\left(e^{+} e^{-}\right)$which is crucial in searching for the BSM signal mediated by $h^{\prime}$. We will only consider the scenario with all particles on shell given by the mass hierarchy: $m_{h^{\prime}}>2 m_{A^{\prime}}, m_{A^{\prime}}>2 m_{e}$. In this conditions the kinematic is maximally different with respect to $e^{+} e^{-} \rightarrow 3\left(e^{+} e^{-}\right) \mathrm{SM}$ process, and the $h^{\prime}$ decays always prompt.

The physics of the two processes suggest that, in particular at low energy, the BSM process (signal) will produce a non negligible contribution to the total cross section. In fact the $\mathrm{SM} e^{+} e^{-} \rightarrow 3\left(e^{+} e^{-}\right)$cross section (background) grows as $\sim\left(\log \frac{s}{m_{e}^{2}}\right)^{4}$ while the Higgs-strahlung cross sections drops as $1 / s$, resulting in a signal over background scaling:

$$
\frac{S}{B} \approx \frac{m_{e}^{2}}{s\left(\log \frac{s}{m_{e}^{2}}\right)^{4}} .
$$




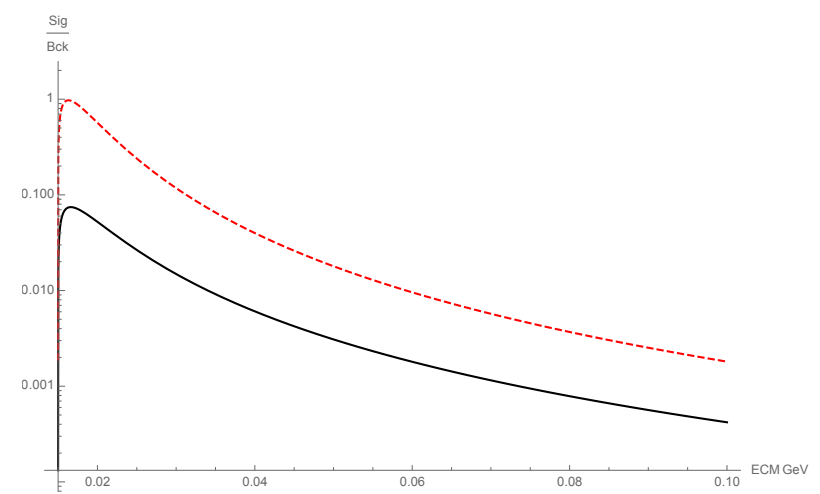

Figure 6. Black line: S/B ratio using Leading Log EPA approximation. Red dashed line: S/B ratio using numerical EPA approximation.

As we already pointed out in section 3, due to accidental cancellation the LLEPA approximation grossly overestimates the $e^{+} e^{-} \rightarrow 3\left(e^{+} e^{-}\right) \mathrm{SM}$ cross section, which is not dominated by the $\log ^{4}$ term, and therefore the S/B scaling will actually be smaller than naively expected using eq. (4.5).

Nevertheless we also observed that the actual value of the $e^{+} e^{-} \rightarrow 3\left(e^{+} e^{-}\right)$cross section is much smaller than expected, providing a much higher starting value of the S/B ratio. The ratio $\sigma_{h^{\prime} A^{\prime}} / \sigma_{6 e}$ is shown in figure 6 for center of mass energies ranging from $0.02 \mathrm{GeV}$ to $0.1 \mathrm{GeV}$ setting $m_{h^{\prime}}=10 \mathrm{MeV}, m_{A^{\prime}}=5 \mathrm{MeV}, \epsilon^{2}=1 \times 10^{-6}$ and $\alpha_{D}=0.1$ in eq. (4.4).

The value of $\epsilon^{2}$ has been chosen small enough to evade experimental constraints coming from visibile dark photon searches [13]. It's interesting to point out that, after the new precise calculation of $\sigma_{e^{+}} e^{-} \rightarrow 3\left(e^{+} e^{-}\right)$proposed in this work, the $\mathrm{S} / \mathrm{B}$ ratio (red line) is increased by roughly one order of magnitude compared to previous estimates (black line). Up to a CM energy $\sim 30 \mathrm{MeV}$ the contribution from $h^{\prime}$ to the total cross section exceeds $10 \%$, and will therefore be observable even by just measuring the total $e^{+} e^{-} \rightarrow 3\left(e^{+} e^{-}\right)$ cross section. When all the particle are on mass shell the value of the $\lambda$ factors in eq. (4.4) varies just by small factors changing the values of $m_{h^{\prime}}$ and $m_{A^{\prime}}$ having it's maximum for $m_{h}^{\prime} \sim m_{A^{\prime}}$.

\subsection{Background suppression}

In this section we would like to point out that appropriate experimental cuts on the final $e^{+} e^{-} \rightarrow 3\left(e^{+} e^{-}\right)$state can strongly enhance the $\mathrm{S} / \mathrm{B}$ ratio. Let us begin by noticing that the distribution of the virtual photons emitted by the initial positron and electron is peaked in the region of small energy and small angle. This can be seen from eq. (A.4) which behaves like $\sim \frac{d \boldsymbol{k}_{\perp}^{2}}{\boldsymbol{k}_{\perp}^{2}} \frac{d x}{x}, x$ being the fraction of the photon's energy and $\boldsymbol{k}_{\perp}$ the photon's transverse momentum with respect to the parent electron (or positron). This implies that the initial electron (positron) emits a final electron (positron) which is collinear and has about the same energy of the initial electron (positron). 
In other words, most of the final events are characterised, in the c.m. frame, by a pair electron-positron emitted back to back and with an invariant mass close to the initial c.m. energy $\sqrt{s}$. This signature is very different from that of the signal event: in this case two mass shell bosons ( $h^{\prime}$ and $A^{\prime}$ ) are created and, broadly speaking, the energy is evenly distributed between the final $e^{+} e^{-}$pairs. Moreover, three $e^{+} e^{-}$pairs invariant masses reconstruct the $A^{\prime}$ mass.

In the case of PADME things happen in the lab frame, and in this case we will have in most of the events a final positron collinear to the beam and carrying almost all of the available energy. Also in this case then, we expect the signal signature to be very different from the background one. To precisely compute the kinematical distributions of the events in PADME goes beyond the scope of the present paper, but it should be clear that such a study can only result in a S/B ratio better than the one shown in figure 6 .

\section{Multi lepton perspectives in PADME}

In this section we try to derive the possible perspective for observing multi lepton final states and eventually constrain the $h^{\prime}$ parameter space with the PADME experiment at the INFN Laboratori Nazionali di Frascati $[14,15]$. In present literature no observation are reported for $e^{+} e^{-} \rightarrow 3\left(e^{+} e^{-}\right)$or $e^{+} e^{-} \rightarrow 2\left(e^{+} e^{-}\right)$processes and no cross section is available which makes their observations appealing.

PADME is a positron on target experiment with a $\sim 500 \mathrm{MeV}$ energy positron beam impinging on a diamond target. Due to the low beam energy PADME has the opportunity to search for dark higgs in the mass region from few to $15 \mathrm{MeV}$, very difficult to achieve for high energy colliders due to the overwhelming associated $e^{+} e^{-} \rightarrow 3\left(e^{+} e^{-}\right)$background. The experiment is currently taking data for Run II at the DA $\Phi$ NE linac and aims to collect $\sim 1 \times 10^{13}$ positron on target collisions. PADME is a fixed target experiment with a diamond target of $d=100 \mu \mathrm{m}$. The experiment luminosity can be computed using the formula:

$$
L_{\text {inst }}=\frac{I_{b}}{e} N_{A} \frac{Z \rho d}{A}
$$

The relevant quantities are $Z=6$, density of diamond, $\rho=3.51 \mathrm{~g} / \mathrm{cm}^{3}$, and $A=12.01 \mathrm{~g}$ carbon's gram-molecular weight. The $I_{b} / e$ during PADME Run II, corresponds to 49 bunches/s of $\sim 27000$ positrons leading to $1.3 \times 10^{6}$ positrons on target per second. The resulting instantaneous luminosity of the experiment is $\sim 1.4 \times 10^{-8} \mathrm{pb} / \mathrm{s}$. Considering one year of data taking corresponding to $1 \times 10^{7} \mathrm{~s}$ of effective experiment's running time, the integrated luminosity will be $\sim 0.14 \mathrm{pb}^{-1} / \mathrm{Y}$. According to the ratio of figure 6 , at the CM energy PADME is operating $\left(\sqrt{2 m_{e} E_{\text {Beam }}} \sim 22 \mathrm{MeV}\right)$, the existence of an $h^{\prime}$ will produce very strong enhancement of the $\mathrm{SM} e^{+} e^{-} \rightarrow 3\left(e^{+} e^{-}\right)$cross section even with $\epsilon^{2}$ values as small as $10^{-6}$. To estimate production rates of $e^{+} e^{-} \rightarrow 3\left(e^{+} e^{-}\right)$and $h^{\prime}$ at PADME, we can use eq. (4.4) and eq. (3.3) to compute the actual values of the signal and background cross sections at $E_{\text {Beam }}=500 \mathrm{MeV}$.

- The Dark Higgs production cross section $\sigma_{h^{\prime}} A^{\prime} \sim 1000 \mathrm{pb}$ : one year of PADME data taking with the instantaneous luminosity of Run II will allow to produce $\sim 150 \mathrm{~h}^{\prime}$ mediated $e^{+} e^{-}$in six leptons final states. 
- $\sigma_{e^{+} e^{-} \rightarrow 3\left(e^{+} e^{-}\right)} \sim 1500 \mathrm{pb}$ : one year of PADME data taking with the instantaneous luminosity of Run II will allow to produce $\sim 200 \mathrm{SM} e^{+} e^{-} \rightarrow 3\left(e^{+} e^{-}\right)$events.

Even a modest BG rejection obtained with the present PADME veto system, will allow the dark sector to become the dominant process. A crucial aspect to understand the experimental acceptance, due to minimum track momentum in the PADME spectrometer $(\sim 50 \mathrm{MeV})$, will be to derive the momentum distributions of tracks coming from the two concurring processes, which we are at present not able to derive for $e^{+} e^{-} \rightarrow 3\left(e^{+} e^{-}\right)$. Nevertheless we expect that a small fraction of $e^{+} e^{-} \rightarrow 3\left(e^{+} e^{-}\right)$event will have a minimum track energy higher than $50 \mathrm{MeV}$, reducing their acceptance with respect to $h^{\prime}$ mediated $6 \ell$ final states.

Important information about the dynamic of the $e^{+} e^{-} \rightarrow 3\left(e^{+} e^{-}\right)$process could be derived from the observation of $e^{+} e^{-} \rightarrow 2\left(e^{+} e^{-}\right)$. A first evidence of this process was obtained in the early ' 70 at Laboratori Nazionali di Frascati [16] without being able to obtain a cross section measurement. In this case the cross section is much higher $\left(\sim \alpha^{2}=\right.$ 10000), as it is the expected to be the experimental acceptance due to higher average track momentum. Computing the cross section value with eq. (2.3) at PADME energies we get $1.2 \times 10^{8} \mathrm{pb}$ leading to order $10^{7} e^{+} e^{-} \rightarrow 2\left(e^{+} e^{-}\right) / \mathrm{y}$ at PADME. Even with a small experimental acceptance PADME has the possibility of observing for the first time this process at energies below $1 \mathrm{GeV}$ already in Run II data.

The dominant backgrounds to the measurement of SM multi-lepton final states at PADME are the pile-up of multiple Bhabha scattering processes, and the beam related background. The very high instantaneous rate of positrons on target during PADME Run II $\left(\sim 100 e^{+} / \mathrm{ns}\right)$ can lead to the simultaneous occurrence of 2 or more Bhabha scattering. The background contribution coming from the pile of multiple Bhabha scattering, can be estimated using data by measuring the rates of $4(6)$ tracks with a total energy $\sim 2(3)$ times the beam energy. Preliminary studies also demonstrated that the total energy cut is very effective in rejecting this background source, being the total energy at least twice as much as for genuine multi-leptons events. Concerning beam related background, PADME is routinely acquiring data sets with no target to study beam background characteristics. Applying the 4(6) leptons selection on this dedicated data sets the beam background contribution can be directly estimated.

\section{Conclusions}

In the present paper we revised current EPA based calculations of the SM cross section for the processes $e^{+} e^{-} \rightarrow 2\left(e^{+} e^{-}\right)$and $e^{+} e^{-} \rightarrow 3\left(e^{+} e^{-}\right)$, in order to compare them to possible dark sector contributions. We pointed out that, due to accidental cancellations, the Leading Log approximation produces a gross over estimate of the cross section at energies below $10 \mathrm{GeV}$, relevant for both fixed target experiments and collider one like Belle II. We confirmed, using complete tree level calculation and improved analytic EPA based calculations, the results for the $e^{+} e^{-} \rightarrow 2\left(e^{+} e^{-}\right)$obtained by authors of [7] with a full EPA based calculation. Using our new approach we have been able to derive for the 
first time an analytic cross section of $e^{+} e^{-} \rightarrow 3\left(e^{+} e^{-}\right)$including all the logarithmic terms. Comparing the result with the Leading Log EPA approximation we discovered that the SM cross section is up to 1 order of magnitude lower with respect to previous estimates. Concerning dark sector contributions, as consequence, we observed that the contribution of $h^{\prime}$ mediated $6 \ell$ final state its dominant at low energies, even for small values of the $\epsilon$ mixing parameter. The potential in measuring for the first time the $e^{+} e^{-} \rightarrow 2\left(e^{+} e^{-}\right)$ and $e^{+} e^{-} \rightarrow 3\left(e^{+} e^{-}\right)$cross section and constraining the dark sector observable with the PADME experiment is also explored.

\section{Acknowledgments}

We would like to thank the director of the Laboratori Nazionali di Frascati Dr. F. Bossi for encouraging this work from the early stage, and Dr. F. Giacchino for useful discussion on the numerical calculations.

\section{A The Equivalent Photon Approximation (EPA)}

In this paper we consider QED processes of the kind $e^{+} e^{-} \rightarrow e^{+} e^{-} X$, where $X=e^{+} e^{-}$ or $X=e^{+} e^{-} e^{+} e^{-}$. These processes are hard to calculate even at tree level, especially the second one that features a huge number of Feynman diagrams and a six-body phase space; in fact no tree level analytical or numerical calculation is available for this process. It is however possible to use the Equivalent Photon Approximation, whose origin dates back to a long time ago [19-21], that has been used also in the context of weak interactions [22], and that in the case of strong interactions is better known as Altarelli-Parisi equations for the evolution of structure functions [23]. In this appendix we describe this approximation and estimate the uncertainty with which the cross sections of our interest can be calculated.

In order to understand EPA, it is crucial to observe that the photon propagators become singular when the emitted photon is collinear with emitting electron. Indeed, emitting indices for the sake of clarity and defining $k=(\omega, \boldsymbol{k}), P=(E, \boldsymbol{p}), P^{\prime}=\left(E^{\prime}, \boldsymbol{p}^{\prime}\right)$, $\boldsymbol{p} \cdot \boldsymbol{k}=|\boldsymbol{p}||\boldsymbol{k}| \cos \theta$ we have: ${ }^{2}$

$$
k^{2}=2 m^{2}-2 E E^{\prime}+2 E E^{\prime} \sqrt{1-\frac{m^{2}}{E^{2}}} \sqrt{1-\frac{m^{2}}{E^{\prime 2}}} \cos \theta
$$

where $m$ is the electron mass. Since we are interested in the limit $E, E^{\prime} \gg m$, we can Taylor expand (A.1) in the small parameter $m^{2}$, obtaining:

$$
-k^{2}=-2 E E^{\prime}(1-\cos \theta)+\left(\frac{E}{E^{\prime}} \cos \theta+\frac{E^{\prime}}{E} \cos \theta-2\right) m^{2}+\mathcal{O}\left(m^{4}\right)
$$

As $\theta \rightarrow 0$ the first term in the expansion drops down and $-k^{2}$ is of the order of the small parameter $m^{2}$. If also $m$ goes to 0 , then the singularity is exposed since $\frac{1}{k^{2}} \rightarrow \infty$. In other terms, $m$ cuts off the $\theta=0$ singularity; for this reason this is called a 'mass singularity'. In

\footnotetext{
${ }^{2}$ Note that $-k^{2}$ is positive defined, i.e. $k^{2}<0$.
} 


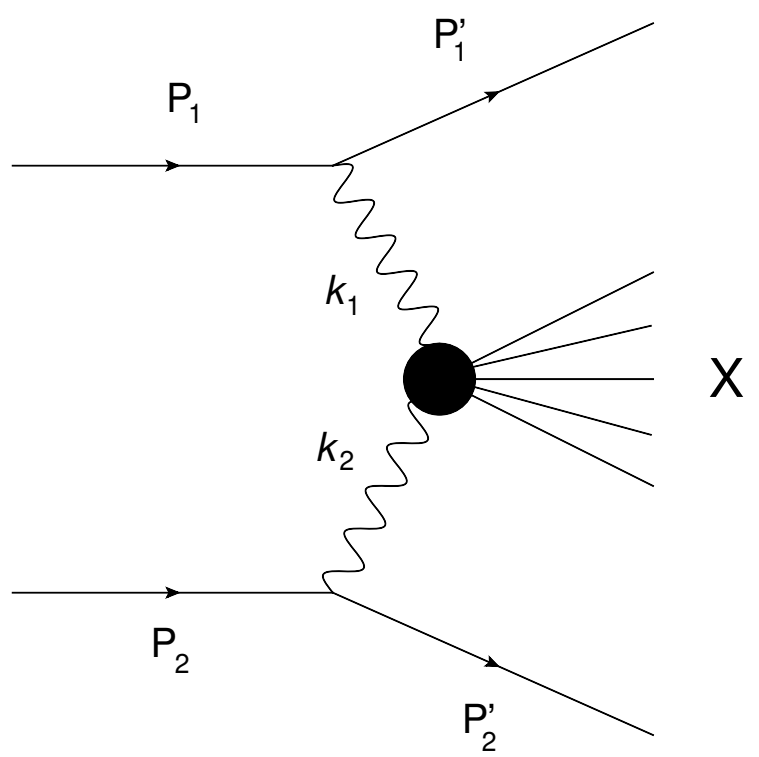

Figure 7. Diagram of the Equivalent Photon Approximation for the process $e^{+} e^{-} \rightarrow e^{+} e^{-} X$, where $X$ is a generic multiparticle state. An electron $\left(P_{1}\right)$ and a positron $\left(P_{2}\right)$ emit one photon each; the photons scatter through the process $\gamma\left(k_{1}\right) \gamma\left(k_{2}\right) \rightarrow X$. In this paper we consider $X=e^{+} e^{-}$ and $X=e^{+} e^{-} e^{+} e^{-}$.

any case, the presence of this singularity enhances the contribution of the EPA diagram of figure 7 with respect to the other diagrams contributing to the process; from the discussion above it is clear that the enhancement grows bigger and bigger as the c.m energy of the process grows, so that the EPA approximation becomes a better one at high energies. In the following we shall see that the enhancement is logarithmic, i.e. proportional to $L=\log \frac{E}{m}$.

In the region that gives the largest contribution, which is the collinear region, where the emitted photon is (almost) collinear to the emitting electron, we can write $k=x P+\boldsymbol{k}_{\perp}$. Here $x$ is the fraction of the electron momentum carried by the gluon, while $\boldsymbol{k}_{\perp}$ is the component of $\boldsymbol{k}$ perpendicular to $\boldsymbol{p}$. For the reasons stated above, $\boldsymbol{k}_{\perp}$ is 'small', $\left|\boldsymbol{k}_{\perp}\right| \ll E$. With these notations, the Equivalent Photon Approximation then amounts to writing (see figure 7):

$$
\sigma\left(e^{+} e^{-} \rightarrow e^{+} e^{-} X ; s\right)=\int d x_{1} d x_{2} f_{\gamma e}\left(x_{1}\right) f_{\gamma e}\left(x_{2}\right) \sigma\left(\gamma \gamma \rightarrow X ; Q^{2}=x_{1} x_{2} s\right)
$$

where $s$ is the invariant mass of the initial state, while $Q^{2}$ is the invariant mass of the $\gamma \gamma$ pair. There are a few simplifying hypotheses underlying the EPA, namely:

- Eq. (A.3) is a probabilistic model in which the emission of photons with distribution $f_{\gamma e}\left(x_{i}\right)$ is factorized with respect to the photons scattering. However, in order to do the computation, one should consider all the relevant Feynman diagrams, add them including interferences. The rationale behind the simplified expression is that the contribution in figure 7 is enhanced by the two photon propagators, as discussed above.

- The $\sigma\left(\gamma \gamma \rightarrow X ; Q^{2}=x_{1} x_{2} s\right)$ cross section is calculated with onshell photons $\left(k_{i}^{2}=\right.$ 0 ) while the photons are virtual in the diagram. Moreover the invariant mass of 
the subprocess $\gamma \gamma \rightarrow X$ is calculated as $Q^{2}=x_{1} x_{2}$, thus neglecting the transverse components of the photons' momenta.

While it is hard in general to assess the precision with which the EPA approximates the 'true' value for the cross section, at the end of this section we estimate such a precision for the processes of relevance in this paper.

The structure function $f_{\gamma e}$ can be calculated in QED to give $[17,18]$ :

$$
f_{\gamma e}(x)=\frac{\alpha}{2 \pi} \int_{\left(\boldsymbol{k}_{\perp}^{2}\right)_{\min }}^{\left(\boldsymbol{k}_{\perp}^{2}\right)_{\max }} \frac{d \boldsymbol{k}_{\perp}^{2}}{\boldsymbol{k}_{\perp}^{2}} P_{\gamma e}(x) ; \quad P_{\gamma e}(x)=\frac{1+(1-x)^{2}}{x}
$$

Notice that one can apply the approximations leading to (A.3), (A.4) to very different physical situations. For instance, provided the energy is sufficiently high, the electron can emit a weak gauge boson instead of a photon [22]. In this case one talks about Equivalent Boson Approximations and the changes with respect to the QED case considered here are: the substitution of $\alpha$ with the weak coupling constant $\alpha_{W}$ and the appearance of a structure function for longitudinal bosons, absent in the case of photons. ${ }^{3}$ The initial particle can be for instance a proton instead of an electron, and one then has distributions for quarks and gluons. The $P_{g q}$ Altarelli Parisi splitting function where $g=$ gluon and $q=$ quark is, in fact, identical to the $P_{\gamma e}(x)$ appearing in (A.4). There are, however, a couple of notable differences between QED and QCD. In the case of QED, the extrema $\left(\boldsymbol{k}_{\perp}^{2}\right)_{\min },\left(\boldsymbol{k}_{\perp}^{2}\right)_{\max }$ are determined by kinematics and this allows for a calculation of $f_{\gamma e}$, as we show below. In the case of QCD however, while $\left(\boldsymbol{k}_{\perp}^{2}\right)_{\max }$ is of the order of the invariant mass $Q^{2}$ of the subprocess, $\left(\boldsymbol{k}_{\perp}^{2}\right)_{\text {min }}$ is given by an infrared cutoff $\mu^{2}$ of the order of the scale $\Lambda_{\mathrm{QCD}}$ below which the perturbative calculation is senseless due to uncalculabel nonperturbative effects. The integration over $\boldsymbol{k}_{\perp}^{2}$ gives is then $\log \frac{Q^{2}}{\mu^{2}}$ and depends on the arbitrary scale $\mu$ so that $f_{g q}$ is not perturbatevely calculable in QCD. Nevertheless, what (A.4) provides is an information on the dependence of the structure function on the $Q^{2}$ of the process. Then, one can measure a given structure functions at a 'low' scale $Q_{0}^{2}$ and use this equation to determine the structure function at a 'high' scale $Q^{2}$. This task is performed by the Altarelli-Parisi equations, that are an all-order extension of (A.4) in the form of integro-differential equations that take into account large logaritms $\log \frac{Q^{2}}{Q_{0}^{2}}$ to all orders in perturbation theory. And here is another difference with the EPA approach, which considers only terms of the first order in $\alpha$.

Getting back to the EPA, since the 'partonic' cross section $\sigma_{p}(\gamma \gamma \rightarrow X)$ depends on the combination $x_{1} x_{2}$, it is convenient to change variables $x_{1}, x_{2} \rightarrow x=x_{1}, \tau=x_{1} x_{2}$ in (A.3), obtaining;

$$
\sigma(s)=\int_{\epsilon}^{1} d \tau \mathcal{L}(\tau) \sigma_{p}(\tau s) \quad \mathcal{L}(\tau)=\int_{\tau}^{1} \frac{d x}{x} f_{\gamma e}(x) f_{\gamma e}\left(\frac{\tau}{x}\right)
$$

where $\epsilon$ is defined in terms of the threshold invariant mass $M_{t h}^{2}$ of the partonic subprocess; for instance in the case of $\gamma \gamma \rightarrow e^{+} e^{-}$we have $\epsilon \equiv \frac{M_{t h}^{2}}{s}=\frac{4 m^{2}}{s}$. In the case of the processes

\footnotetext{
${ }^{3}$ The ElectroWeak case for more general cases has been studied in [24].
} 
considered in this paper, a careful examination of kinematical bounds entails:

$$
f_{\gamma e}(x)=\frac{\alpha}{2 \pi} \log \frac{1}{x^{2}} \Rightarrow \mathcal{L}(\tau) \approx \frac{2 \alpha^{2}}{3 \pi^{2}} \frac{\left(\log \frac{1}{\tau}\right)^{3}}{\tau}
$$

where the value of the luminosity $\mathcal{L}$ has been obtained by fetching $f_{\gamma e}(x)$ into (A.5) and neglecting terms of order $\left(\log \frac{1}{\tau}\right)^{2}$ (leading $\log$ approximation). Using this luminosity and the known expression for $\sigma(\gamma \gamma) \rightarrow e^{+} e^{-}[7]$ we obtain:

$$
\sigma\left(e^{+} e^{-} \rightarrow e^{+} e^{-} e^{+} e^{-}\right) \approx \frac{28 \alpha^{4}}{27 \pi m^{2}}\left(\log \frac{s}{m^{2}}\right)^{3}
$$

so that the leading behaviour of the cross section is a growth like $L^{3}=\left(\log \frac{s}{m^{2}}\right)^{3}$. The situation is different for $e^{+} e^{-} \rightarrow 3\left(e^{+} e^{-}\right)$: the luminosity is the same, but the 'partonic' cross section $\gamma \gamma \rightarrow 4$ leptons is approximately constant, differently from the case of $\gamma \gamma \rightarrow 4$ leptons where the cross section decreases like $1 / Q^{2}$ for high $Q^{2}$. We obtain:

$$
\sigma_{2 e \rightarrow 6 e}(s) \approx \frac{\alpha^{2}}{6 \pi^{2}} l^{4} \sigma_{\gamma \gamma \rightarrow 4 e}=\frac{\alpha^{6} l^{4}}{3 \pi^{3} m^{2}} \frac{175 \zeta(3)-38}{72} \approx 0.79 \frac{\alpha^{6} l^{4}}{\pi^{3} m^{2}}
$$

where the value for $\sigma_{\gamma \gamma \rightarrow 4 e}$ has been taken from [12].

Open Access. This article is distributed under the terms of the Creative Commons Attribution License (CC-BY 4.0), which permits any use, distribution and reproduction in any medium, provided the original author(s) and source are credited.

\section{References}

[1] B. Batell, M. Pospelov and A. Ritz, Probing a secluded U(1) at B-factories, Phys. Rev. D 79 (2009) 115008 [arXiv:0903.0363] [inSPIRE].

[2] R. Essig, P. Schuster and N. Toro, Probing dark forces and light hidden sectors at low-energy $e^{+} e^{-}$colliders, Phys. Rev. D 80 (2009) 015003 [arXiv:0903.3941] [INSPIRE].

[3] H.-B. Li and T. Luo, Probing dark force at BES-III/BEPCII, Phys. Lett. B 686 (2010) 249 [arXiv: 0911.2067] [INSPIRE].

[4] M. Hostert and M. Pospelov, Novel multi-lepton signatures of dark sectors in light meson decays, arXiv:2012.02142 [INSPIRE].

[5] BaBAr collaboration, Search for low-mass dark-sector Higgs bosons, Phys. Rev. Lett. 108 (2012) 211801 [arXiv:1202.1313] [INSPIRE].

[6] Belle collaboration, Search for the dark photon and the dark Higgs boson at Belle, Phys. Rev. Lett. 114 (2015) 211801 [arXiv: 1502.00084] [INSPIRE].

[7] V.M. Budnev, I.F. Ginzburg, G.V. Meledin and V.G. Serbo, The two photon particle production mechanism. Physical problems. Applications. Equivalent photon approximation, Phys. Rept. 15 (1975) 181 [INSPIRE].

[8] A. Accardi et al., e+ @JLab white paper: an experimental program with positron beams at Jefferson lab, arXiv:2007.15081 [INSPIRE].

[9] BABAR collaboration, Search for a narrow resonance in $e^{+} e^{-}$to four lepton final states, arXiv:0908.2821 [INSPIRE]. 
[10] A. Belyaev, N.D. Christensen and A. Pukhov, CalcHEP 3.4 for collider physics within and beyond the Standard Model, Comput. Phys. Commun. 184 (2013) 1729 [arXiv:1207.6082] [INSPIRE].

[11] J. Alwall et al., The automated computation of tree-level and next-to-leading order differential cross sections, and their matching to parton shower simulations, JHEP 07 (2014) 079 [arXiv: 1405.0301] [INSPIRE].

[12] H. Cheng and T.T. Wu, Photon-photon scattering close to the forward direction, Phys. Rev. D 1 (1970) 3414 [INSPIRE].

[13] M. Battaglieri et al., US cosmic visions: new ideas in dark matter 2017: community report, arXiv: 1707.04591 [INSPIRE].

[14] M. Raggi and V. Kozhuharov, Proposal to search for a dark photon in positron on target collisions at DAФNE Linac, Adv. High Energy Phys. 2014 (2014) 959802 [arXiv: 1403.3041] [INSPIRE].

[15] M. Raggi, V. Kozhuharov and P. Valente, The PADME experiment at LNF, EPJ Web Conf. 96 (2015) 01025 [arXiv: 1501.01867] [InSPIRE].

[16] C. Bacci et al., Gamma-gamma interaction processes at adone $e^{+} e^{-}$storage ring. Measurement of the reaction $e^{+} e^{-} \rightarrow e^{+} e^{-} e^{+} e^{-}$, Lett. Nuovo Cim. 3S2 (1972) 709 [INSPIRE].

[17] Y.L. Dokshitzer, Calculation of the structure functions for deep inelastic scattering and $e^{+} e^{-}$annihilation by perturbation theory in quantum chromodynamics, Sov. Phys. JETP 46 (1977) 641 [Zh. Eksp. Teor. Fiz. 73 (1977) 1216] [INSPIRE].

[18] V.N. Gribov and L.N. Lipatov, Deep inelastic ep scattering in perturbation theory, Sov. J. Nucl. Phys. 15 (1972) 438.

[19] E. Fermi, Über die Theorie des Stoßes zwischen Atomen und elektrisch geladenen Teilchen, Z. Phisik 29 (1924) 315.

[20] C.F. von Weizsacker, Radiation emitted in collisions of very fast electrons, Z. Phys. 88 (1934) 612 [INSPIRE].

[21] E.J. Williams, Correlation of certain collision problems with radiation theory, Kong. Dan. Vid. Sel. Mat. Fys. Med. 13N4 (1935) 1.

[22] S. Dawson, The effective W approximation, Nucl. Phys. B 249 (1985) 420.

[23] G. Altarelli and G. Parisi, Asymptotic freedom in parton language, Nucl. Phys. B 126 (1977) 2988.

[24] P. Ciafaloni and D. Comelli, Electroweak evolution equations, JHEP 11 (2005) 022 [hep-ph/0505047] [INSPIRE]. 